\title{
Which soft lens power is better for piggyback in keratoconus? Part II
}

\author{
Miguel Romero-Jiménez ${ }^{\mathrm{a}, *}$, Jacinto Santodomingo-Rubido ${ }^{\mathrm{b}}$, \\ Jose-Manuel González-Meijóme ${ }^{\mathrm{c}}$, Patricia Flores-Rodriguez ${ }^{\mathrm{d}}$, Cesar Villa-Collar ${ }^{\mathrm{e}}$ \\ a MRG Doctores S.C, Madrid, Spain \\ ${ }^{\mathrm{b}}$ Menicon REDIC, Menicon Co., Ltd, Japan \\ ${ }^{c}$ Clinical E' Experimental Optometry Research Lab, Center of Physics, University of Minho, Braga, Portugal \\ ${ }^{\mathrm{d}}$ Pontificia Universidad Católica de Valparaíso, Chile \\ e European University of Madrid, Spain
}

\section{A R T I C L E I N F O}

\section{Article history:}

Received 17 February 2014

Received in revised form

18 September 2014

Accepted 26 September 2014

\section{Keywords:}

Piggyback

Contact lens

Cornea

Keratoconus

Keratometry

\begin{abstract}
A B S T R A C T
Purpose: To evaluate how soft lens power affects rigid gas-permeable (RGP) lens power and visual acuity (VA) in piggyback fittings for keratoconus.

Methods: Sixteen keratoconus subjects (30 eyes) were included in the study. Piggyback contact lens fittings combining Senofilcon-A soft lenses of $-6.00,-3.00,+3.00$ and $+6.00 \mathrm{D}$ with Rose K2 RGP contact lenses were performed. Corneal topography was taken on the naked eye and over each soft contact lens before fitting RGP lenses. Mean central keratometry, over-refraction, RGP back optic zone radius (BOZR) and estimated final power as well as VA were recorded and analyzed.

Results: In comparison to the naked eye, the mean central keratometry flattened with both negative lens powers ( $p<0.05$ in all cases), did not change with the +3.00 soft lens power $(p=1.0)$; and steepened with the +6.00 soft lens power $(p=0.02)$. Rigid gas-permeable over-refraction did not change significantly between different soft lens powers (all $p>0.05$ ). RGP's BOZR decreased significantly with both positive in comparison with both negative soft lens powers (all $p<0.001$ ), but no significant differences were found among negative- or positive-powers separately (both $p>0.05$ ). Estimated RGP's final power increased significantly with positive in comparison with negative lens powers (all $p<0.001$ ), but no significant differences were found among negative or positive lens powers separately (both $p>0.05$ ). Visual acuity did not change significantly between the different soft lens powers assessed (all $p>0.05$ ).

Conclusion: The use of negative-powered soft lenses in piggyback fitting reduces RGP lens power without impacting VA in keratoconus subjects.
\end{abstract}

(C) 2014 British Contact Lens Association. Published by Elsevier Ltd. All rights reserved.
Rigid gas-permeable (RGP) contact lenses represent the most common and successful management option for early to moderate cases of keratoconus [1], despite the development of surgical treatments that aim to regularize the anterior corneal surface (i.e. intrastromal corneal ring implantation) [2] or to stabilize the progression of the disease (i.e. cross-linking) [3]. Currently, a number of contact lens designs and materials are available to fit irregular corneas, particularly keratoconus as it is the most common primary ectasia $[1,4]$. Corneal RGP [5,6], semi-scleral [7,8], scleral [9], hybrid [10] or custom-made soft contact lenses [11,12] are commonly used to manage keratoconus patients. However, corneal RGP lenses are probably the most widely prescribed contact lens design

* Corresponding author. Current address: Vallehermoso 32, Bajo A, Madrid, Spain. Tel.: +34 62 8782201; fax: +34 915919737.

E-mail address: mrjlentes@gmail.com (M. Romero-Jiménez). in keratoconus subjects worldwide. Discomfort or recurrent anterior corneal surface erosions are sometimes associated with the use of these lenses leading to reduced wearing time and sometimes to discontinuation from lens wear. In cases of unbearable discomfort with RGP lens wear, a soft lens can be used as a carrier of the RGP lens; the fitting of a RGP lens onto a soft contact lens is known as piggyback system and was first described by Baldone in the early 1970 s $[13,14]$. It is estimated that piggyback is used by about $2 \%$ of keratoconus contact lens wearers [15]. Furthermore, VA is similar with piggyback systems in comparison to RGP lens wear alone [16].

Piggyback fitting is normally recommended using a low-positive powered soft contact lens because it shifts the highest elevation of the cornea to a more centered location and hypothetically facilitates RPG lens centration, although the use of a negative-powered lens was recommended by Baldone in steeper corneas [17]. However, despite of the latter and although central keratometry is steeper in keratoconus in comparison to normal corneas, most 
practitioners continue using a low positive-powered soft lens in piggyback fittings for the keratoconic cornea $[18,19]$. In a previous study, we demonstrated that negative-powered soft lenses create a flatter and less powerful anterior corneal surface, which reduces coma-like aberration potentially leading to an improvement in visual acuity, thus making them more suitable for piggyback contact lens fittings [20]. However, the results of that study were devired FROM using a theoretical approach as no RGP lenses were fitted over soft contact lenses of different powers. Furthermore, as a result of the latter, we were unable to assess the impact of negative-powered soft lenses on visual acuity or RGP lens fitting (i.e. centration, movement and power). On the contrary, in a very recent study performed on regular corneas, the use of a low positive-powered soft contact lens was recommended for piggyback fitting in all cases (i.e. regular and irregular corneas) because: (1) it is believed to facilitate RGP lens centration; and (2) it does not contribute to the total power of the piggyback lens system [21].

Based in all the above, the purpose of the present study was to evaluate whether positive- or negative-powered soft lenses are better for piggyback in keratoconus with regards to RGP lens fitting and visual acuity.

\section{Methods}

This was a prospective non-dispensing masked study. Sixteen subjects ( 30 eyes) of at least 18 years of age with keratoconus were enrolled. A comprehensive optometric and ophthalmologic examination was performed in all cases which included: uncorrected and corrected visual acuity with contact lenses, biomicroscopy examination, fundus evaluation, keratometry and corneal topographic analysis (Pentacam Eye Scanner, software version 1.16.r:04, Oculus Inc, Wetzlar, Germany). The patients should be habitual RGP lens wearers to be enrolled. Exclusion criteria were previous history of acute corneal hydrops, corneal surgery or other ocular disease. All subjects were provided written informed consent to participate in the study. The study followed the Tenets of the Declaration of Helsinki and was approved by the Institutional Review Board of MGR Doctores Ophthalmology Clinic, Madrid, Spain.

The soft contact lenses used in the study were made of Senofilcon A material and all had BOZRs of $8.40 \mathrm{~mm}$ (Johnson \& Johnson Vision Care Inc., FL, USA). RGP lens fitted in this study were Rose K2 manufactured in tisilfocon A material (Menicon Co., Ltd. Nagoya, Japan). In subjects with bilateral keratoconus, the study was performed on both eyes as it is well established keratoconus is a bilateral and asymmetric condition [22,23].

\subsection{Fitting method}

Corneal topography was obtained in the naked eye (i.e. without contact lens) in eligible subjects. Then, one of the soft lenses were fitted and allowed to settle down on the eye for $10 \mathrm{~min}$ before taking a new corneal topography with the soft lens over the cornea (Fig. 1). Then, a RGP contact lens was fitted over the soft contact lens with a BOZR equal to the mean central keratometry measured over the soft lenses minus $0.2 \mathrm{~mm}$, accordingly to the manufacturer's fitting guide. Then, the BOZR was flattened or steepened in $0.10 \mathrm{~mm}$ steps until the first definite apical clearance lens (FDACL) was observed following previous reported methodology $[5,24]$. Once the FDACL was achieved, the BOZR was flattened $0.10 \mathrm{~mm}$ to obtain a threepoint-touch fitting approach [5]. The RGP lens was allowed to settle on the eye for 30-45 min and the lens fitting was assessed using low weight molecular fluorescein (Haag-Streit, Koeniz, Switzerland). The soft lens was required to cover the entire cornea without overpassing the limbus, whereas the RGP lens must be well centered whithin the limbal area. A RGP lens was considered well centrated when the pupil was covered by the lens' optic zone without touching the limbus on primary gaze (Fig. 2). Rigid gas-permeable lens movement was required to be between 0.5 and $1.5 \mathrm{~mm}$ with blink. When RGP lens centration and/or movement were inappropriate, changes to the edge lift were performed to improve fitting (i.e. excessive movement or upper lens decentration was remedied by decreasing the edge lift, whereas insufficient movement or downward lens decentration was solved increasing the edge lift). Once an optimal lens fitting was achieved, an over-refraction was performed. The procedure described above was repeated with soft lenses of $-6.00,-3.00,+3.00$ and $+6.00 \mathrm{D}$, consecutively. The same investigator (M.R.-J.) carried out all the lens fittings and assessments.

\subsection{Statistical analysis}

Differences in mean central keratometry, RGP over-refraction, RGP's BOZR and RGP final estimated power as well as best contact lens visual acuity (BCLVA), between the naked eye and the different soft lenses were analyzed using a repeated measures analysis of variance (ANOVA) followed by post-hoc tests, if necessary. Equality of variances and sphericity were tested using the Levene and Mauchly tests, respectively, to select appropriate $p$-values. Statistical analyses were performed using SPSS 15.0 software (SPSS Inc, Chicago, IL, USA). The level of statistical significance was taken as $5 \%$.

\section{Results}

Eleven males (68.8\%) and 5 females (31.3\%) habitual corneal RGP contact lens wearers with keratoconus were included in the study. The mean age ( \pm SD; range) of the subjects was $34.9 \pm 8.8$ (range 21.3-49.4) years. Thirty eyes (16 right eyes and 14 left eyes) were fitted. According to the keratoconus severity score [25], 21 eyes and 9 eyes had mild and moderate keratoconus, respectively. No subjects were previously fitted using a piggyback contact lens system.

An average of $2.55 \pm 0.78$ RGP trial lenses were necessary to achieve an optimal piggyback lens fit. No clinically significant differences on RGP lens centration or movement were found between different soft lens powers fitted in this study (Fig. 2).

Significant differences were found in mean central keratometry between the different conditions tested $(p<0.001)$. In comparison to the naked eye, the mean central keratometry flattened with negative-powered soft lenses $(p<0.05$ with -3.00 and -6.00 soft lenses); did not change with the +3.00 soft lens power $(p=1.0)$; and steepened with the +6.00 soft lens power $(p=0.02)$ (Table 1$)$.

Although RGP over-refraction difference between -6.00 and +6.00 soft lenses was 3.26 D, being higher (more negative) with both positive lenses in comparison with both negative lenses (Table 1), such differences were not statistically significant ( $p=0.91$, Table 2 ).

Statistically significant differences were found in RGP's BOZRs fitted on top of each of the 4 different soft contact lenses $(p<0.001)$. The BOZRs were steeper with both positive power lenses in comparison with both negative power lenses (Table 1). However, the BOZR was not statistically different among negative power $(-3.00$ vs $-6.00 \mathrm{D}$ ) or positive power $(+3.00 \mathrm{vs}+6.00 \mathrm{D}$ ) lenses (Table 2 ). The more positive the soft contact lenses fitted, the smaller the differences between mean central keratometry and BOZR (Fig. 3).

Statistically significant differences were also found in estimated RGP's final powers ( $p<0.001$, Fig. 4 ). The estimated final power of the RGP lens increased significantly with both positive power lenses in comparison with both negative power soft lenses (all $p<0.001$ ), but no significant differences were found among negative- or positive-powers separately (both $p>0.05$ ). 


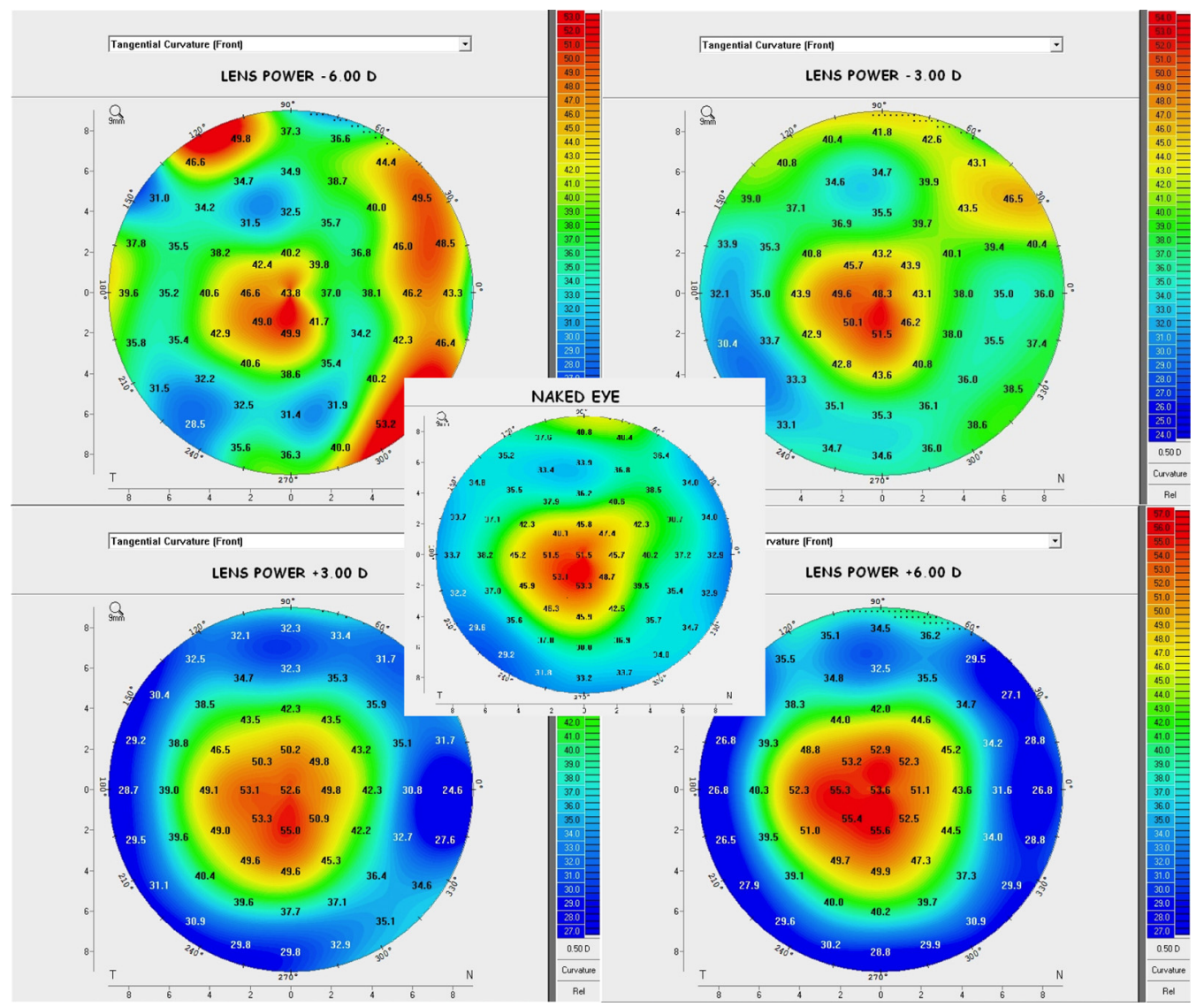

Fig. 1. Changes in anterior corneal topography with soft contact lenses of different powers. Cone size increases with positive-powered soft lenses and decreases with negative-powered lenses.

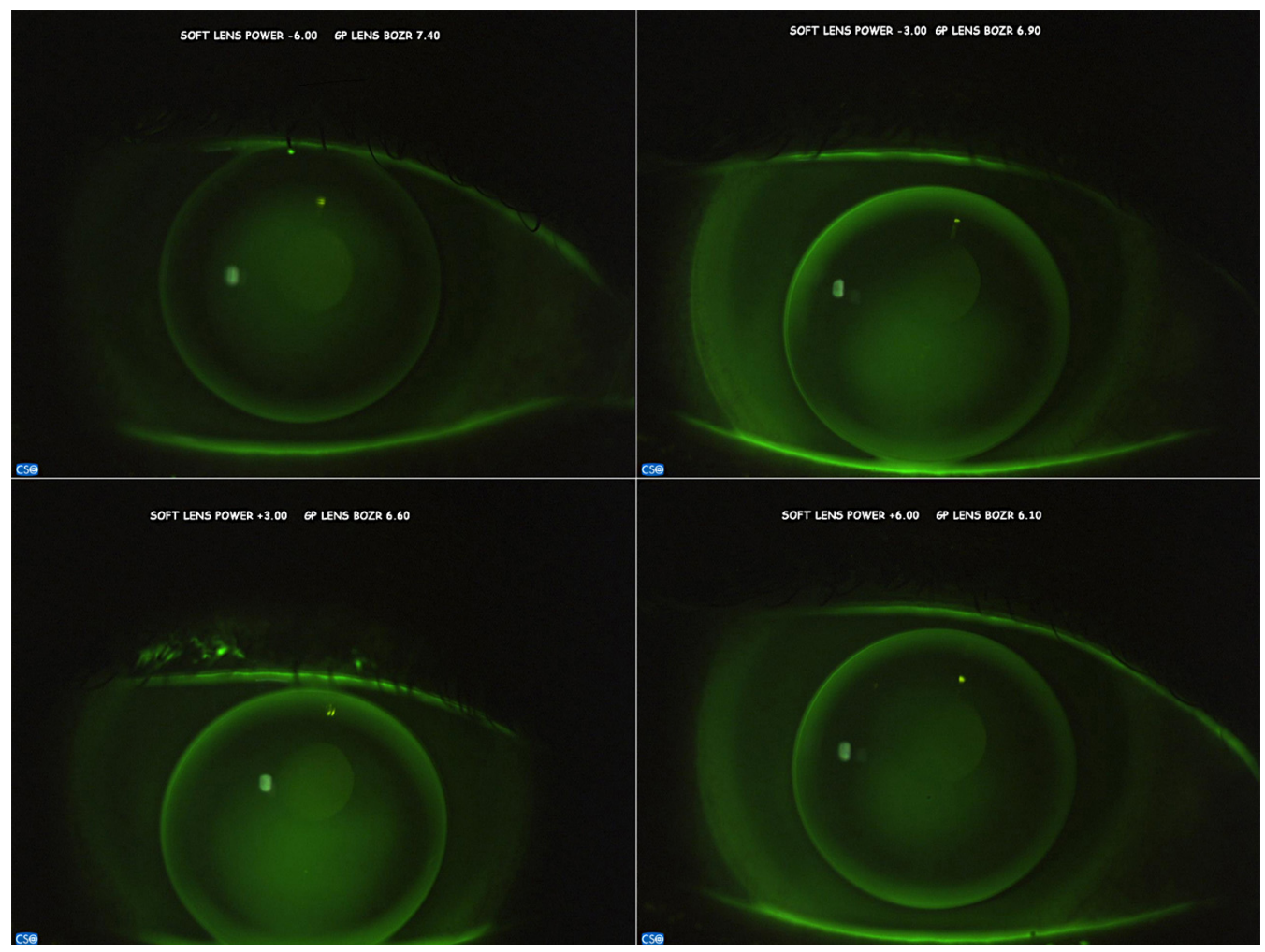

Fig. 2. Piggyback lens system's flourograms of soft contact lenses of different powers. All images show the first definite apical clearance lens for each soft lens power. 
Table 1

Mean $( \pm$ SD) keratometric, back optic zone radius, over refraction and visual acuity measures over the naked eye and over soft contact lenses of different powers.

\begin{tabular}{|c|c|c|c|c|}
\hline Measure & $\operatorname{MCK}(\mathrm{mm})$ & RGP over Rx (D) & RGP lens' BOZR (mm) & VA log MAR \\
\hline Naked & $6.83 \pm 0.80$ & - & - & - \\
\hline$-6.00 \mathrm{D}$ & $7.75 \pm 0.77$ & $0.05 \pm 5.35$ & $7.54 \pm 0.66$ & $0.07 \pm 0.10$ \\
\hline$-3.00 \mathrm{D}$ & $7.32 \pm 0.75$ & $-1.44 \pm 5.19$ & $7.22 \pm 0.62$ & $0.06 \pm 0.08$ \\
\hline $3.00 \mathrm{D}$ & $6.69 \pm 0.66$ & $-3.10 \pm 5.51$ & $6.64 \pm 0.50$ & $0.06 \pm 0.08$ \\
\hline $6.00 \mathrm{D}$ & $6.42 \pm 0.62$ & $-3.31 \pm 5.90$ & $6.38 \pm 0.37$ & $0.05 \pm 0.08$ \\
\hline
\end{tabular}

MCK, mean central keratometry; RGP, rigid gas-permeable; BOZR, back optic zone radius; Rx, refraction; VA, visual aquity.

Table 2

Statistical analyses between soft lenses ( $p$-values).

\begin{tabular}{|c|c|c|c|c|c|c|c|}
\hline \multirow[t]{2}{*}{ Measure } & \multirow[t]{2}{*}{ ANOVA $p$-value } & \multicolumn{3}{|c|}{ Post-hoc $p$-values for $-6.00 \mathrm{D}$ lens } & \multicolumn{2}{|c|}{$\begin{array}{l}\text { Post-hoc } p \text {-values } \\
\text { for }-3.00 \mathrm{D} \text { lens }\end{array}$} & \multirow{2}{*}{$\begin{array}{l}\text { Post-hoc } p \text {-values } \\
\text { for }+3.00 \mathrm{D} \text { lens } \\
+6.00\end{array}$} \\
\hline & & -3.00 & +3.00 & +6.00 & +3.00 & +6.00 & \\
\hline RGP final estimated power & $<0.001$ & 1.0 & $<0.001$ & $<0.001$ & 0.019 & $<0.001$ & 1.0 \\
\hline BOZR & $<0.001$ & 0.176 & $<0.001$ & $<0.001$ & $<0.001$ & $<0.001$ & 0.505 \\
\hline
\end{tabular}

RGP, rigid gas-permeable; BOZR, back optic zone radius.

Best corrected visual acuity (Table 1 ) did not change significantly between the different soft lens powers employed $(p=1.0)$.

\section{Discussion}

The fitting of piggyback contact lens systems in keratoconus is challenging for eye care practitioners because it requires a wide knowledge of different contact lens designs and materials, as well as of the keratoconic corneal shape. This study aimed at understanding how soft lens power affects the fitting of an RGP lens on a piggyback system, particularly taking into account that most practitioners are likely to use low positive soft lens powers in piggyback fittings in keratoconus. In fact, the latter has been recommended in a very recent study performed on regular corneas [21]. However, the central keratometry of keratoconus subjects is much steeper than that of normal subjects and thus the results obtained in normal corneas might not be extrapolated for fittings carried out on irregular corneas. In fact, we found similar RGP centration and movement for all soft lens powers assessed in this study using a three-point-touch fitting approach and the FDACL concept as a first step to optimize the fitting $[5,24]$.

We have found that both negative power soft lenses flatten the mean central keratometry and $+6.00 \mathrm{D}$ soft lens power steepens the mean central keratometry, which it is in agreement with previous findings [18,20,21]. Therefore, fitting negative power soft contact lenses in piggyback for keratoconus provides a flatter and less

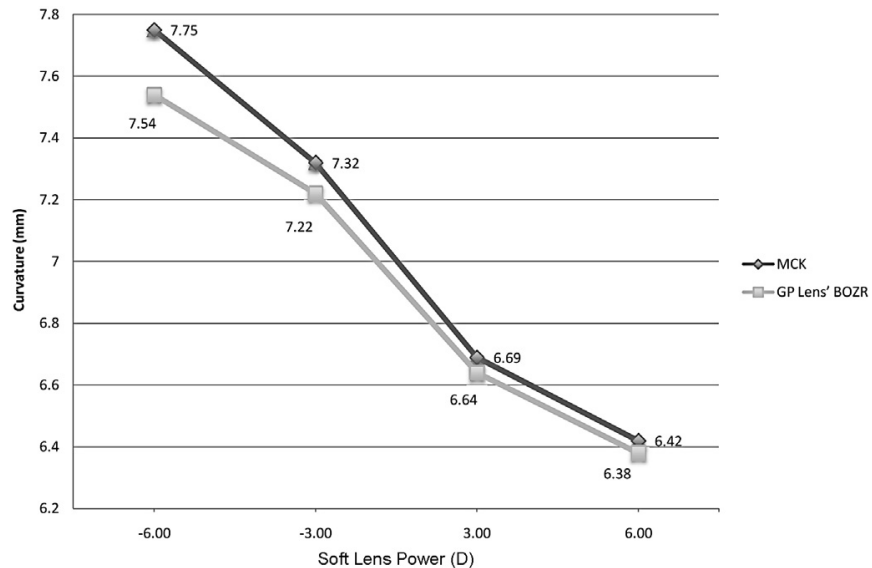

Fig. 3. Comparison of mean central ekratometry and final rigid gas-permeable lens' back optic zone radius. powerful anterior corneal surface, which necessarily will impact on piggyback lens system's power.

The absence of differences found in RGP over-refraction was due to the RGP lens' design. Rose K2 contact lens trial lenses features a variable power related to the BOZR (i.e. as the BOZR increases, the power decreases and vice versa). In the present study, the latter might be considered as an advantage because the RGP trial lenses used were very similar to the final lenses that would have been neeed to be dispensed, which allowed a reliable fitting evaluation with regards to lens movement and centration.

Rigid gas-permeable's BOZR became steeper the more positive the soft lens power used, which, as is explained below, impacts on RGP lens' power and weight and thus on the on-eye lens stabilization and flexure. The difference between lens BOZR and mean central keratometry was greater with both negative power soft lenses in comparison with both positive power lenses. This was because the cone becomes wider with positive soft lenses in comparison with negative soft lenses (Fig. 1). In fact, the fitting of soft positive contact lenses it creates a bowl that mimics better the central back surface of the Rose K2 lens and at the same time, contributes to center better the highest point of the cornea, which is typically inferior in keratoconus corneas. The latter could be interpreted as it would be easier to fit a RGP lens over a positive-powered soft lens in comparison with negativepowered soft lens. However, the modern contact lens designs for keratoconus (i.e. Rose K2) allows easy modifications on lens' edge lift, which facilitates the control of lens' movement and centration.

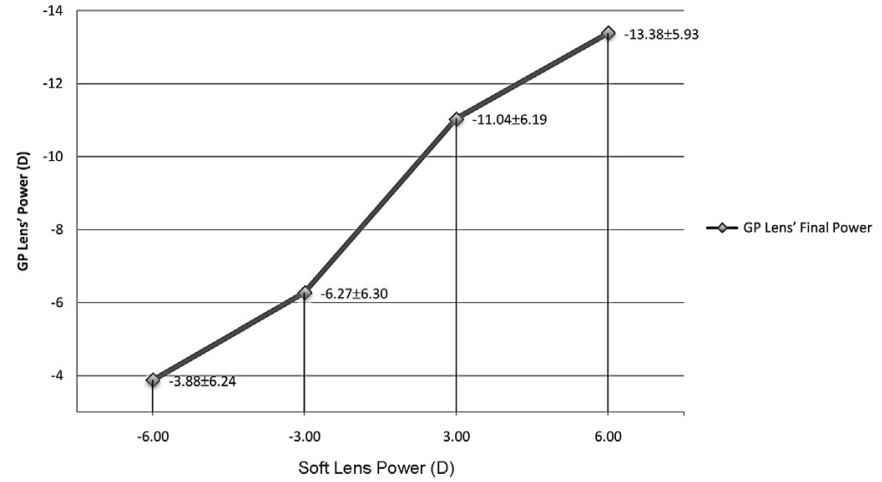

Fig. 4. Estimated final power of the rigid gas-permeable lens. The difference between $-6.00 \mathrm{D}$ and $+6.00 \mathrm{D}$ lenses is $9.5 \mathrm{D}$. 
The RGP lens' final estimated power was significantly more negative with positive-powered soft lenses in comparison with negative-powered lenses, which was expected due to the variation of the mean central keratometry and the subsequent lens' BOZR steepening. It has been recently reported that only $21 \%$ of labelled soft lens power is transferred to the piggyback lens system for -6.00 and +6.00 soft lens power in regular corneas [21]. Thus, theoretically, a difference of $2.5 \mathrm{D}$ should be found on the RGP lens final estimated power in the present study between soft lens powers of -6.00 and +6.00 . However, RGP final estimated power was, on average, $-9.5 \mathrm{D}$ more negative when fitted over a +6.00 soft lens in comparison to with a -6.00 soft lens. This is explained by the modification of the mean central keratometry caused by fitting negative or positive soft lenses. The flattening on the effective anterior corneal surface caused by a mild negative soft lens leaded to a decrease on the effective refractive power of the ocular system. When a RGP lens was fitted over negative-powered soft lenses, a flatter BOZR was necessary to achieve an optimal lens fit in comparison to the lens' BOZR fitted over positive-powered soft lenses, which leaded to a reduction on RGP lens power. On the other hand, for the same overall lens diameter, the greater the lens' power (negative or positive), the heavier the lens. A heavier lens is likely to decenter more on the cornea with normal blink in comparison to a lighter lens. Furthermore, RGP lens' central thickness becomes thinner when negative power increases. In the case of the Rose K2 lens design, the central thickness varies from $0.17 \mathrm{~mm}$ in a lens of $7.55 \mathrm{~mm}$ of BOZR to $0.13 \mathrm{~mm}$ in a lens of $6.40 \mathrm{~mm}$ of BOZR (manufacturer's data). It has been previously reported that the thinner the center of a RGP lens, the greater the lens flexure and thus the induction of residual astigmatism [26]. Additionally, using a high minus carrier together with a lower minus RGP lens would enable the optic zone of the RGP to be larger, which is likely to reduce flare in dim light conditions Thus, a less powerful RGP lens would decrease the likelihood of lens flexure, residual astigmatism and flare leading to an improvement of visual quality.

In the present study, best-corrected visual acuity was similar with the different combinations of soft contact lens powers and RGP lenses tested. However, the use of negative- or positive-powered contact lenses might impact high order aberrations of anterior corneal surface. In fact, it has been previously reported that high order aberrations, especially spherical- and coma-like aberrations are greater in keratoconus in comparison with normal subjects [27]. Furthermore, positive-powered soft lenses induce positive spherical aberration whereas negative-powered soft lenses induce negative spherical aberration with, the greater the lens power, the higher the spherical- aberration induced [28]. In keratoconus, spherical-like aberration is normally positive in sign. Our group has previously reported a significant reduction in coma-like aberration with the use of negative-powered soft lenses in comparison to positive-powered soft contact lenses fitted in keratoconus eyes [20]. Awwad and co-workers [28] also found a significant reduction in spherical aberration with the use of negative-powered soft lenses in comparison to positive-powered soft lenses. Thus, the use of negative-powered lenses in piggyback lens fitting in keratoconus is likely to reduce spherical-like and coma-like high order aberrations, which are probably associated to an improvement in visual quality.

An additional issue to consider is the fact that negative lenses will provide higher oxygen transmissibility to the piggyback system at the center of the cornea [29,30]. This might be important for better oxygen availability around the cone. The corneal epithelium, which is usually affected from its basal membrane to the more superficial cells, might benefit from this strategy from a physiological point of view.
In conclusion, despite low positive-powered soft contact lenses have been traditionally used for piggyback fitting in keratoconus, the results of this study as well as our previous study [24] confirms that the use of a mild negative-powered soft contact lenses allows the fitting of a flatter and less powered RGP lens. The lighter weight of an RGP lens is likely to result in better on-eye lens centration and movement. In addition, a more negative soft lens might reduce sphericaland coma-like aberrations leading to an improvement of visual quality. Finally, a negative lens will provide an overall better performance in terms of oxygen transmissibility at the center of the piggyback system. Considering all these factors into account, the use of a low negative-powered silicone hydrogel contact lens for piggyback in keratoconus might be more appropriate to use in comparison to low positive-powered contact lenses.

\section{References}

[1] Romero-Jiménez M, Santodomingo-Rubido J, Wolffsohn J. Keratoconus: a review. Contact Lens Anterior Eye 2010;33:157-66.

[2] Colin J, Malet FJ. Intacts for the correction of keratoconus: two-year follow-up. J Cataract Refract Surg 2007;33:69-74.

[3] Caporossi A, Mazzotta C, Baiocchi S, Caporossi T. Long-term results of rivoflavin ultraviolet a corneal collagen cross-linking for keratoconus in Italy: the Siena eye cross study. Am J Ophthalmol 2010;149:585-93.

[4] Ortenberg I, Behrman S, Geraisy W, Barequet IS. Wearing time as a measure of success of scleral lenses for patients with irregular astigmatism. Eye Cont Lens 2013;39:381-4.

[5] Romero-Jiménez M, Santodomingo-Rubido J, González-Méijome JM. An assessment of the optimal fit rate in keratoconus subjects using three-point-touch and apical touch fitting approaches with the Rose K2 lens. Eye Contact Lens 2013;39:269-72

[6] Tse JS. Piggy-back contact lens fitting using intra-limbal RGP lens in a Chinese patient with rapid keratoconic changes: a case report. Contact Lens Anterior Eye 2011;34(Suppl. 1):39-40.

[7] Romero-Jiménez M, Flores-Rodríguez P. Utility of a semi-scleral contact lens design in the management of the irregular cornea. Contact Lens Anterior Eye 2013;36:146-50

[8] Schornack MM, Patel SV. Scleral lenses in the management of keratoconus. Eye Contact Lens 2010;36:39-44.

[9] Visser ES, Visser R, van Lier HJ, Otten HM. Modern scleral lenses part I: clinical features. Eye Contact Lens 2007;33:13-20.

[10] Carracedo G, González-Méijome JM, Lopes-Ferreira D, Carballo J, Batres L. Clinical performance of a new hybrid contact lens for keratoconus. Eye Cont Lens 2013;40:2-6.

[11] González-Méijome JM, Peixoto-de-Matos SC, Queiros A, Jorge JM, Diaz-Rey A. Quality if vision with spectacles, special silicone hydrogel and gas permeable contact lens in keratoconic patients. Int J Kerat Ect Cor Dis 2013;2: 56-9.

[12] Carballo-Alvarez J, Puell MC, Cuiña R, Diaz-Valle D, Vazquez JM, Benitezdel-Castillo JM. Soft contact lens fitting after intrastromal corneal ring segment implantation to treat keratoconus. Contact Lens Anterior Eye 2014;37: 377-81.

[13] Baldone JA, Clark WB. Contact lenses in the aphakic child. Contact lens Med Bull 1970;3:25.

[14] Baldone JA. The fitting of hard lenses onto soft contact lenses in certain diseased conditions. Contact lens Med Bull 1973;6:15

[15] Zadnik K, Barr JT, Edrington TB, Everett DF, Jameson M, McMahon TT, et al. Baseline findings in the Collaborative Longitudinal Evaluation of Keratoconus (CLEK) Study. Invest Ophthalmol Vis Sci 1998;39:2537-46.

[16] Woo GC, Callender MG, Egan DJ. Vision through corrected keratoconic eyes with two contact lens systems. Int Contact Lens Clin 1984;11:748-56.

[17] Baldone JA. Piggy-back fitting of contact lenses. CLAO J 1985;11(April-June (2)):130-4.

[18] O'Donnell C, Codina CM. A hyper-Dk contact lens system for keratoconus. Eye Contact Lens 2004;30:44-8.

[19] Sengor T, Kurna SA, Aki S, Özkurt Y. High Dk piggyback system for contact lens-intolerant keratoconus patients. Clin Ophthalmol 2011;5:331-5.

[20] Romero-Jiménez M, Santodomingo-Rubido J, González-Méijome JM. Which soft lens power is better for piggyback fitting in keratoconus? Contact Lens Anterior Eye 2013;36:45-8.

[21] Michaud L, Brezau D, Corbeil ME. Contribution of soft lens of various powers to the optics of a piggy-back system on regular corneas. Contact Lens Anterior Eye 2013;36:318-23

[22] Zadnik K, Steger-May K, Fink BA, Joslin CE, Nichols JJ, Rosenstiel CE, et al Between-eye asymmetry in keratoconus. Cornea 2002;21:671-9.

[23] Chopra I, Jain AK. Between-eye asymmetry in keratoconus in an Indian population. Clin Exp Ophthalmol 2005;88:146-52. 
[24] Edrington TB, Barr JT, Zadnik K, Davis LJ, Gundel RE, Libassi DP, et al, Standardized rigid contact lens fitting protocol for keratoconus. Optom Vis Sci 1996;73:369-75.

[25] McMahon TT, Szczotka-Flynn L, Barr JT, Anderson RJ, Slaughter ME, Lass JH et al. A new method for grading the severity of keratoconus: the keratoconus severity score (KSS). Cornea 2006;25:794-9.

[26] Collins MJ, Frankiln R, Carney LG, Bergiel C, Lagos P, Chebib D. Flexure of thin rigid contact lens. Contact Lens Anterior Eye 2001;24:59-64.

[27] Negishi K, Kumanomido T, Utsumi Y, Tsubota K. Effect of higher-order aberrarions on visual function in keratoconic eyes with a rigid gas permeable contact lens. Am J Ophthalmol 2007;144:924-9.
[28] Awwad ST, Sanchez P, Sanchez A, Mcculley JP, Cavanagh HD. A preliminary in vivo assessment of higher-order aberrations induced by a silicone hydrogel monofocal contact lens. Eye Contact Lens 2008;34:2-5.

[29] Weissman BA, Ping Y. Calculated tear oxygen under tension contact lenses offering resistance in series: piggyback and scleral lenses. Contact Lens Anterior Eye 2006;29:231-7.

[30] López-Alemany A, González-Méijome JM, Almeida JB, Parafita MA, Refojo MF. Oxygen transmissibility of piggyback system with conventional soft and silicone hydrogel contact lenses. Cornea 2006;25:214-9. 